\title{
A running battle with oral diseases - are we in with a sporting chance?
}

Gallagher J, Ashley P, Petrie A, Needleman I. Oral health-related behaviours reported by elite and professional athletes. Br Dent J 2019; 227: 276-280. https://doi.org/10.1038/s41415-019-0617-8.

\section{Here has been an advertisement.}

SPRINGERNATURE

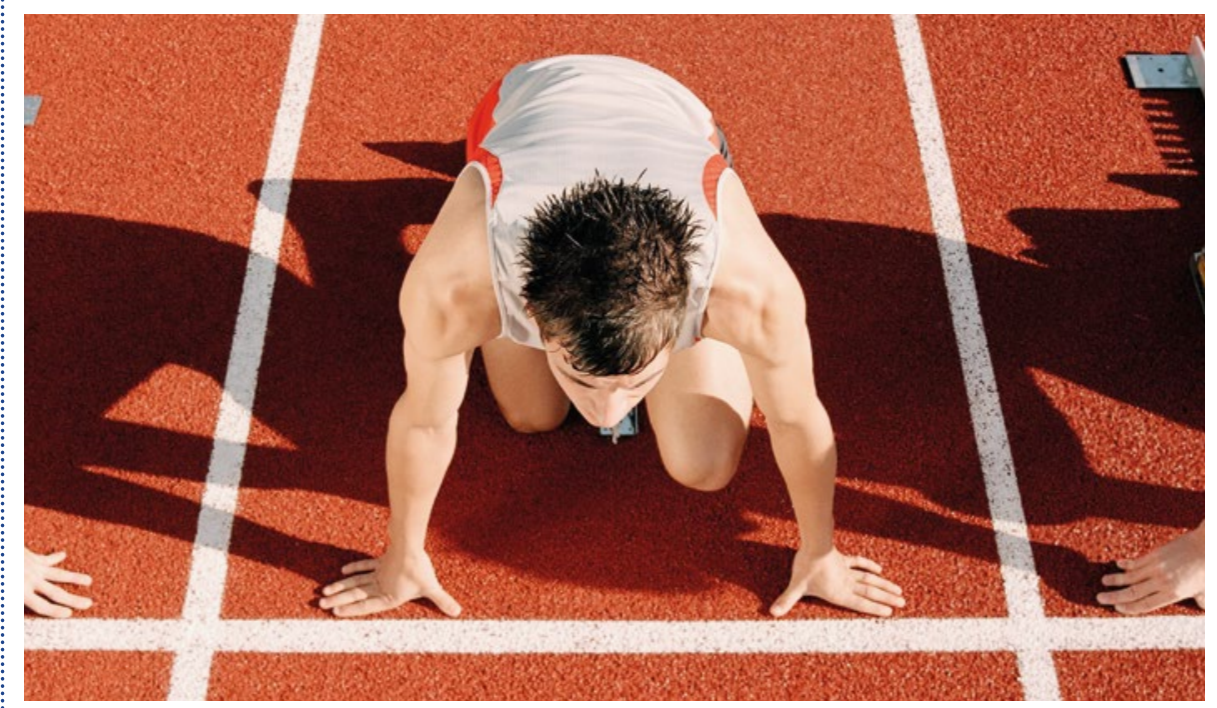

As the 2020 Olympic Games approach, many athletes around the world will be training for the competition. With the high performance demands that these elite sports bring, protecting athletes' health becomes a high priority. Despite this, studies suggest most athletes present with a range of oral diseases including dental caries, periodontal diseases and erosive tooth wear. This paper aims to explore athletes' oral health behaviours, knowledge of the potential risk factors and identifies opportunities to implement behaviour changes.

Since research in this area is minimal, researchers at the Eastman Dental Institute surveyed 344 elite and professional athletes, preparing for the 2016 Olympic Games, using a self-administered questionnaire. The questionnaire, followed oral health screening, quantifying the number of oral diseases prevailing among the group. Athletes were categorised as either 'endurance,' 'mixed/team' or 'strength and power', distinguishing participants from 11 different sport types accordingly.

Predominantly, the results demonstrated favourable oral health behaviours, with as many as $94 \%$ reporting to brush their teeth twice daily and around $44 \%$ using methods of interdental cleaning. However, fewer than half had attended for a dental visit within the preceding six months and from an oral health aspect, the diets detailed were also less encouraging. A large proportion of athletes demonstrated awareness of the impact of sugary foods and drinks on oral health, yet $28 \%$ were considered high consumers of sugar. The majority of athletes consume energy drinks, bars and/or gels during training, implying an increased and inappropriate intake of refined carbohydrates. When questioned about their willingness to reduce their snacking habits, fewer than half confirmed this to be a viable change. Many were open to making adjustments to their diet or oral hygiene routine and attending dental visits more routinely.

Although largely positive oral health behaviours were reported, the incidence of oral diseases did not reflect this and closely compared to those observed in the general population in England, Wales and Northern Ireland. Ultimately, this research could be critical in designing effective intervention strategies towards improving the oral health of athletes, thus reducing potential performance impacts. It is likely that the most effective methods of delivering oral health promotion are achievable when embedded within general health promotion. There is a degree of fragility where the oral health of elite and professional athletes is concerned. Perhaps clinicians should endeavour to address the individual behaviours of athletes in a more personalised approach and provide the motivation to make changes that improve their overall performance.

By Jay Patel

Dental Student, University of Leeds $\frac{\text { 产 }}{\text { () }}$ 